\title{
Artificial Induction of Labor with Misoprostol: Maternal and Fetal Prognosis at the Referral Health Center of Commune I of the District of Bamako
}

\section{Mamadou Bakary Coulibaly ${ }^{*}$, Alassane Traoré ${ }^{1}$, Mody Abdoulaye Camara ${ }^{2}$, Mamadou Sima ${ }^{3}$, Bourama Kané4, Kalba Tembiné5, Ibrahima Tégueté ${ }^{3}$, Youssouf Traoré6}

\author{
${ }^{1}$ Department of Gynecology of Hôpital du Mali, Bamako, Mali \\ ${ }^{2}$ Service of Anesthesia and Resuscitation of Hôpital du Mali, Bamako, Mali \\ ${ }^{3}$ Service of Gynecology-Obstetrics CHU Point G, Bamako, Mali \\ ${ }^{4}$ Service of Pediatric of Hôpital du Mali, Bamako, Mali \\ ${ }^{5}$ Service of Medical Imaging of Hôpital du Mali, Bamako, Mali \\ ${ }^{6}$ Service of Gynecology-Obstetrics CHU Gabriel Toure, Bamako, Mali \\ Email: *mbccolby1@yahoo.com
}

\begin{abstract}
How to cite this paper: Coulibaly, M.B., Traoré, A., Camara, M.A., Sima, M., Kané, B., Tembiné, K., Tégueté, I. and Traoré, Y. (2021) Artificial Induction of Labor with Misoprostol: Maternal and Fetal Prognosis at the Referral Health Center of Commune I of the District of Bamak. Open Journal of Obstetrics and Gynecology, 11, 70-79. https://doi.org/10.4236/ojog.2021.112009
\end{abstract}

Received: December 31, 2020

Accepted: February 5, 2021

Published: February 8, 2021

Copyright $\odot 2021$ by author(s) and Scientific Research Publishing Inc. This work is licensed under the Creative Commons Attribution International License (CC BY 4.0).

http://creativecommons.org/licenses/by/4.0/

\section{(c) (i) Open Access}

\begin{abstract}
Artificial induction of labor (AIL) is the set of techniques intended to induce childbirth in order to reduce maternal-fetal mortality. The aim of this paper was to study the maternal and fetal prognosis after the artificial induction of labor with misoprostol. It was a descriptive prospective study conducted from September 1, 2018 to February 28, 2019 at the maternity unit of the Referral Health Center (RHC) in commune I of Bamako. It was about any pregnant woman at term who had received misoprostol as part of the artificial induction of labor. 102 cases of artificial induction with misoprostol $200 \mu \mathrm{g}$ were collected out of 3641 deliveries, or a frequency of $2.8 \%$. The age group $28-32$ years accounted for $56.86 \%$ with an average age of 28.8 years. Multiparous represented $54.90 \%$ of the number. The history of diabetes and hypertension involved $37.25 \%$ of the pregnant women. The main indication was premature rupture of membranes (PRM) (40.2\%). Induction was performed between the $37^{\text {th }}$ and $41^{\text {st }}$ week of amenorrhea (69.6\%). The trigger index according to BISHOP was greater than 7 (50.98\%). Induction was done only with $3 / 4$ of misoprostol tablet $200 \mu \mathrm{g}$ (43.14\%). The average labor time was 7 hours (89.22\%). The vaginal birth concerned $92.16 \%$ of pregnant women. An APGAR10 was noted in $97.85 \%$ of newborns after 05 minutes. AIL with misoprostol was practiced at the Center. The main indication was the premature rupture of the membranes. It improves the maternal and fetal prognosis.
\end{abstract}




\section{Keywords}

Induction, Misoprostol, Maternal and Fetal Prognosis, Commune I

\section{Introduction}

Induction of labor is a common practice in gynecology and obstetrics for various medical grounds. The obstetrician can induce labor, the benefits of which lie in the decrease of maternal and/or perinatal mortality and morbidity as well as in the reduction of the cesarean section rate [1]. Successful induction depends on the initial assessment of the cervix by using mainly the Bishop score [2]. An unfavorable cervix, defined by a score $<7$, exposes to a higher risk of C-section. For these situations, different pharmacological or mechanical methods are available to achieve a preliminary cervical ripen [3]. These different methods can be cervical maturation with prostaglandins (misoprostol), membrane sweeping, the use of oxytocin. Prostaglandins play a basic role and current researches are focused on the mechanisms that lead to their release [4]. The main indications are: the post-term pregnancy, fetal death in utero, adnexal fetal complications, hypertensive and metabolic complications [1].

Induction of labor has become a common obstetric practice around the world [5]. Currently, the induction rate is around $30 \%$ of births in developed countries; $22 \%$ in France according to the national perinatal survey of 2016 [6]. It was $15 \%$ in 2008 in Pakistan [7]; 2.49\% in 2001 in the gynecology and obstetrics department of the hospital "Point G" in Mali [8]. Its frequency had never been evaluated in the gynecology and obstetrics department of the RHC of commune I of the district of Bamako. These reasons led us to initiate this work, which aimed to study the AIL by using misoprostol $200 \mu \mathrm{g}$.

\section{Patients and Method}

This was a descriptive cross-sectional study on the prospective collection of data from September 1, 2018 to February 28, 2019 (six months) at the maternity unit of the Referral Health Center (RHC) of Commune I of Bamako district. It is a level II structure in the health pyramid of Mali, whose primary vocation is mother and child health. Was included in the study, any pregnant woman carrying a mono-fetal pregnancy, at term, and or prolonged, in cephalic presentation having received misoprostol $200 \mu \mathrm{g}$ in the setting of artificial induction of labor (AIL) according to the obstetric indication. We're not included in our study: multiple pregnancies, fetuses in transverse or seated presentation, scarring uteri, non-term pregnancies and cases of induction occurring outside the study period.

The variables studied were: the age of the pregnant woman, medical (hypertension, diabetes), obstetric (prenatal consultation, parity, age of pregnancy, number of fetuses, mode of admission, score of APGAR and Bishop's score (Table 1). 
Table 1. Bishop score.

\begin{tabular}{|c|c|c|c|c|}
\hline Parameters & 0 & 1 & 2 & 3 \\
\hline Cervix dilation & closed & $1-2 \mathrm{~cm}$ & $3-4 \mathrm{~cm}$ & $\geq 5 \mathrm{~cm}$ \\
\hline Cervix erasure & $40 \%-50 \%$ & $40 \%-50 \%$ & $60 \%-70 \%$ & $\geq 80 \%$ \\
\hline Cervix consistency & firm & medium & soft & \\
\hline Cervix position & posterior & Central & Anterior & \\
\hline $\begin{array}{l}\text { Positioning of the fetal } \\
\text { presentation in relation } \\
\text { to the sciatic spines }\end{array}$ & $\begin{array}{c}\text { mobile } \\
(3 \mathrm{~cm} \text { above })\end{array}$ & $\begin{array}{c}\text { started } \\
(2 \mathrm{~cm} \text { above })\end{array}$ & $\begin{array}{c}\text { fixed } \\
(\leq 1 \mathrm{~cm} \text { above })\end{array}$ & $\begin{array}{c}\text { engaged } \\
(1-2 \mathrm{~cm} \text { below })\end{array}$ \\
\hline
\end{tabular}

All pregnant women had received a general consultation to assess the maternal condition. An obstetric examination was performed to define: gestational age, genital status, presentation of the fetus, the number of fetuses, the size of the uterus.

For all pregnant women, the indication for induction and the decision to administer misoprostol were discussed in a team. The AIL began very early in the morning on a working day. Before induction of labor, the Bishop score was assessed in all pregnant women. The Bishop score is one of the key parameters in the prognosis of vaginal delivery. It not only makes it possible to make the prognosis of the trigger but also to guide in the choice of the appropriate method. Thus when the Bishop score is favorable greater than or equal to 7; in general, oxytocin infusion with or without artificial rupture of membranes seems to be the preferred method.

But when it is unfavorable less than 7 , it is often necessary to go through a preliminary stage of cervical ripening using prostaglandins and prostaglandin analogues. In our study we used a prostaglandin analogue which is misoprostol. It was administered vaginally at a dose of $50 \mu \mathrm{g}$ every 8 hours without exceeding 3 doses.

The Bishop score was therefore noted before the first insertion and at each assessment of the cervix.

Pregnant women not admitted to labor after the 3 doses were reassessed.

We have used a pre-established survey sheet thanks to the following documents: obstetrical records with partographs; the birth register; the reference/evacuation register; the newborn transfer register; the register of perinatal death; the operating report register.

The variables studied were: age, marital status, level of education, method of admission, pregnancy and parity, medical and surgical history, maternal pathologies, number of antenatal consultations, term of pregnancy, obstetric examination data, condition of the fetus before induction, indications of labor induction with misoprostol, protocol for applying and monitoring vaginal and oral tablets, dose of misoprostol used, delay in onset of uterine contractions after administration of misoprostol, adverse effects of misoprostol, duration of fetal 
expulsion, duration of labor, route of delivery, causes of failure, type of delivery, maternal and fetal prognosis, postpartum monitoring.

The data were collected from: an individual survey sheet, the delivery register, prenatal consultation records and the labor induction protocol sheet.

Data analysis was done with SPSS software version18. For the distribution calculation, we have used the Chi square statistical test. Any value of the probability of error less than 0.05 were considered significant.

\section{Results}

102 cases of artificial induction were collected out of 3641 recorded deliveries, with a frequency of $2.8 \%$. The age group 28 - 32 years accounted for $56.86 \%$ with an average age of 28.8 years and extremes ranging from 18 years to 37 years. Pregnant women were self-admitted (80.39\%) and referred by peripheral structures (19.61\%). Multiparous represented $54.90 \%$ of the number. The history of diabetes and high blood pressure (HBP) involved $37.25 \%$ of pregnant women and $58.82 \%$ had received an antenatal consultation (ANC) by midwives (maternal characteristics in Table 2). The main indications were: premature rupture of the membranes (PRM) (40.2\%) and the post-term pregnancy (21.57\%) (Table $3)$. The decision of induction was made by gynecologists (52.94\%), general practitioners (31.37\%), and midwives (15.69\%).

Table 2. Maternal characteristics and labor initiation parameters.

\begin{tabular}{|c|c|}
\hline Variables (mean and extremes) & $\mathrm{n}=102$ \\
\hline Middle age (year) & $28.8(28-32)$ \\
\hline Multiparous & $56(54.90 \%)$ \\
\hline Self-admission & $82(80.39 \%)$ \\
\hline Antenatal consultation & $1-2(47.6 \%)$ \\
\hline Gestational age in WA & $37-41 \mathrm{SA}(69.61 \%)$ \\
\hline \multicolumn{2}{|l|}{ Bishop score } \\
\hline Bishop 1 - 4 & $14(13.73 \%)$ \\
\hline Bishop 5 - 6 & $36(35.29 \%)$ \\
\hline \multicolumn{2}{|l|}{ Number of misoprostol dosage } \\
\hline $1^{\text {st }}$ dosage & $8(7.84 \%)$ \\
\hline $2^{\text {nd }}$ dosage & $32(31.37 \%)$ \\
\hline $3^{\text {rd }}$ dosage & $44(43.14 \%)$ \\
\hline $4^{\text {th }}$ dosage & $18(17.65 \%)$ \\
\hline Time to onset of uterine contractions & 7 H 54 (52.94\%) \\
\hline
\end{tabular}


The AIL with misoprostol $200 \mu \mathrm{g}$ was performed between the $37^{\text {th }}$ and $41^{\text {st }}$ weeks of amenorrhea (WA) in $69.61 \%$ of cases based on the Bishop score. This score was less than seven (7) in $49.02 \%$ of cases. In $43.14 \%$ of cases $3 / 4$ ofa misoprostol tablet $200 \mu \mathrm{g}$ was sufficient to induce the onset. Induction of labor occurred between 6 and 12 hours (52.94\%) and the average time was 7 hours (89.22\%). Vaginal delivery concerned $92.16 \%$ of pregnant women and C-section $7.84 \%$ of cases. The main indications for C-section were: acute fetal distress (75\%) and onset obstructed labor (25\%). An APGAR 10 was observed in $97.85 \%$ of newborns after five minutes, and $98.92 \%$ had received neonatal assistance according to the service protocol (Table 4). The immediate postpartum assistance was simple (97.06\%). Fever (21.57\%), vomiting (16.67\%) and chills $(14.71 \%)$ were the main side effects pointed out.

Table 3. Distribution according to the indication of induction of labor.

\begin{tabular}{ccc}
\hline Indication & Number & Percentage \\
\hline PRM & 41 & 40.2 \\
Post-term pregnancies & 22 & 21.57 \\
Diabetes/pregnancy & 18 & 17.65 \\
HBP/pregnancy & 12 & 11.76 \\
Missed abortion & 9 & 8.82 \\
Total & 102 & 100 \\
\hline
\end{tabular}

Table 4. Parameters of newborns at birth.

\begin{tabular}{cc}
\hline Neonatal outcomes & Live newborns $=93$ \\
\cline { 2 - 2 } Average weigh of childbirth & Stillborn $=9$ \\
Apgar & $3250(2200-4650)$ \\
& Apgar $0=9$ (stillborn) \\
$1^{\text {st }}$ minute (Apgar $\left.\geq 7\right)$ & Live newborns $=93$ \\
$5^{\text {th }}$ minute (Apgar 10) & $91(89.22 \%)$ \\
$10^{\text {th }}$ minute (Apgar 10) & $91(97.85 \%)$ \\
Pediatric care & $91(97.85 \%)$ \\
No pediatric care & $92(98.92 \%)$ \\
\hline
\end{tabular}




\section{Discussion}

The limits and the difficulties.

- Some patients have not made the 04 antenatal consultations required as part of the screening for risk factors.

- Lack of appropriate labor induction room. The induction takes place in the single delivery room.

The expected benefits of artificial induction are the decrease in maternal mortality and perinatal morbidity and mortality. There are several pharmacological or mechanical methods to achieve a preliminary cervical uterine ripening. During our study, we had used misoprostol $200 \mu \mathrm{g}$ (prostaglandin), certainly the most convenient, but which can present adverse effects in some pregnant women (chills, vomiting, fever and uterine rupture).

\section{Frequency}

At the end of our study, out of the 3641 deliveries performed; 102 pregnant women had undergone induction of labor with misoprostol, or a frequency of 2.8\%. Our data was comparable to those reported by SANGARE M [8] (1.84\%) and Dolo M [9] (2.1\%) in Mali, but significantly lower than the $9.82 \%$ of Kouam [10] in Cameroon. The frequency of induction varies greatly from one region to another and even from one health facility to another [11]. Despite this notion of significant disparity, the trigger rate is generally higher in European countries. In France, in 2016, it was $22 \%$ according to the national perinatal survey [6].

This difference between European countries and developing countries in terms of induction of labor could be explained by the large number of induction for personal reasons carried out in these countries. However, in our regions, particularly in Mali, AIL is reserved for specific indications (HBP, diabetes, post-term, PRM, etc.).

\section{Age}

The age group 28 - 32 years accounted for $56.86 \%$, with a mean age of 28.8 years. These figures were comparable to those of Dolo $M$ [9] who reported the age group 20 - 34 years $(50.61 \%)$ and an average of 27 years. This strong representativeness of the age group 20 to 34 years was also found out by Sangaré M. [8]. Like him, we thought that this age group corresponded to the period of full genital activity.

Method of admission: Our pregnant women who were self-admitted (80.39\%) and referred by peripheral structures (19.61\%). Dolo M [9] reported that $74 \%$ of his pregnant women had self-reliance and $26 \%$ were referred.

History: Diabetes and high blood pressure affected $37.25 \%$ of our pregnant women. Chiesa M [12] in Gabon reported a lower rate than ours (31.9\%).

Parity: Multiparous accounted for $54.90 \%$ of our sample and nulliparous (2.94\%). These proportions were the opposite of Nadia [13] in Tunisia who reported $9.82 \%$ of multiparous and $52 \%$ of nulliparous. Dolo M [9] reported $32 \%$ 
of multiparous.

Antenatal consultations: ANC were performed in $58.82 \%$ by midwives and $21.57 \%$ by gynecologists. We did not find any document of ANC in $19.61 \%$ of our pregnant women. On the other hand, Dolo M [9] reported a proportion of $69 \%$ of ANC performed by midwives and $31 \%$ by gynecologists. In the series of Chiesa $M$ [12] and Nadia O [13], all the pregnancies were monitored by gynecologists.

Indications: Our main indications for labor induction were respectively: PRM (40.2\%); the post-termpregnancy (21.57\%); diabetes (17.65\%); HBP (11.76\%); the missed abortion $8.82 \%$. Chiesa M [12] reported: PRM (47\%); HBP during pregnancy (31.9\%); the missed abortion (9.7\%); post-term pregnancy $(6.9 \%)$ and triggering of convenience $(2.8 \%)$. Nadia $\mathrm{O}$ [13] reported the following rates: post-term pregnancy (48\%), gestational diabetes $(22.7 \%)$, oligohydramnios (18\%) and HBP (11.4\%). Dolo M [9] found out: the post-term pregnancy (35.8\%); PRM (27.15\%); missed abortion (11.25\%); HBP (17.28\%). We found that all of these indications were generally comparable except for a few small differences.

The authors of indication for labor induction: the indication for labor induction had been decided by gynecologists $(52.94 \%)$, general practitioners (31.37\%), and midwives (15.69\%). In the series of Bartusevicius A [11], Chiesa $\mathrm{M}$ [12], and Nadia $\mathrm{O}$ [13], all triggering decisions were made by the gynecologists.

Gestational age: Induction of labor was practiced between 37 - 41 WA in $69.61 \%$ of our pregnant women. These proportions were comparable to those of Bartusevicius A [11] and Nadia O [13]. In all these studies, labor was induced on a pregnancy: missed, at term, or post-term. However, in the work of Dolo M [9], $30.4 \%$ of pregnant women had undergone induction of labor before 36 weeks of amenorrhea, for the reasons of HBP.

Assessment of the BISHOP score: The trigger index according to BISHOP was less than 7 in $49.02 \%$ of cases. These rates were lower than those of Sangaré M [8], Dolo M [9]; Kouam L [10] who pointed out $87.5 \%$; $68.07 \%$ and $58.45 \%$ respectively. Nadia O [13] had obtained a higher rate of $95.5 \%$ for a BISHOP score less than 7.

The dose of misoprostol: the trigger was driven by misoprostol which is a synthetic analogue of prostaglandins. It is therefore essential to accurately assess the benefit/risk ratio of prescribing this molecule, favoring the dosage associated with the greatest safety. In our series $150 \mu$ g or $3 / 4$ of a misoprostol tablet was sufficient to induce the onset in $43.14 \%$. Our doses of misoprostol were comparable to those of Kouam L [10] and Chiesa M [12] in Cameroon, which was 44.5\% and $42.65 \%$ respectively. In Nadia O's series [13], 1/4 of a tablet induced labor in $84 \%$ of patients whereas in the series of Dolo M [9], 1/2 tablets had induced labor in $90.56 \%$.

Time between administration of misoprostol and onset of contractions: In 
our study, induction of labor occurred between 6 and 12 hours in 52.94\%. This rate is comparable to that of Bartusevicius A [11], who had obtained induction of labor in 50\% for the same time. Dolo M [9], reported a delay of 19-24 hours in $75.47 \%$ of pregnant women. Nadia O [13] obtained a delay of less than 6 hours in $70.5 \%$ of pregnant women.

Time of labor: The average time of labor was 7 hours (89.22\%). Our proportions were comparable to those of Nadia O [13] 6.5 hours (90\%) and Bartusevicius A [11] reported 18 hours (78.5\%).

Our triggers with misoprostol were made on healthy unscarred uterus, as in the studies of SANGARE M [8], Dolo M [9], Bartusevicius A [11] Chiesa M [11] and Nadia O [13]. Other induction methods have been tested as in the series by V.Pez [14], focusing on cervical ripening and induction methods using the single balloon catheter compared to double balloon catheter and prostaglandins. These experiences had not been yet evaluated in Mali.

Delivery routes: vaginal delivery is synonymous with successful induction. This route concerned $92.16 \%$ of pregnant women. Nadia O [13] had pointed out $70.4 \%$ vaginally. Chiesa $M$ [12] reported $93 \%$ of vaginal route. The greatest success rate was obtained by Dolo $M$ [9] (98.75\%). The main indications for C-section were acute fetal distress (75\%) and onset obstructed labor (25\%). Our results were similar to the other studies [8] [9] and [13].

Maternal-fetal prognosis: We did not record any maternal death or uterine rupture. In contrast, Nasreen $\mathrm{Ab}$ [15] had recorded uterine ruptures in his study. Live newborns were generally well with an Apgar greater than or equal to 7 out of 10 by the fifth minute $(97.85 \%)$, which was the expected result when making an indication of induction. This result is comparable to those of Dolo M [9] and Diallo D [16] who found $74.6 \%$ and $88.75 \%$ respectively, with an Apgar score greater than 7 after five minutes.

Side effects related to the use of misoprostol: We observed some side effects: fever (21.57\%), vomiting (16.67\%) and chills (14.71\%). These same findings have been reported in different proportions in the series by Dolo $M$ [9], Bartusevicius A [11], Chiesa M [12].

Conclusion: The use of misoprostol in the induction of labor is a common practice in our department. Its utilization in cervical ripening and in the induction of labor decreases the rate of $\mathrm{C}$-section. The main indications were premature rupture of membranes and post-term. Its usage was not without side effects, hence the need for being careful and a fine grained clinical examination in order to eliminate the contraindications of its use.

Author approval: All authors agree on the submission of this article.

Conflict of interest: There is no conflict of interest.

Consent: For this work we received the consent of the patient, that of the Chief department and Director of Hopital du Mali, as well as the Chief Doctor and the Head of the gynecology-obstetrics service of commune I of the district of Bamako. 


\section{Conflicts of Interest}

The authors declare no conflicts of interest regarding the publication of this paper.

\section{References}

[1] Pennell, C.E., Henderson, J.J., O’Neill, M.J., McChlery, S., McCleery, S., Doherty, D.A., et al. (2009) Induction of Labor in Nulliparous Women with an Unfavorable Cervix: A Randomized Controlled Trial Comparing Double and Single Balloon Catheters and $\mathrm{PGE}_{2}$ Gel. An International Journal of Obstetrics \& Gynecology, 116, 1443-1452. https://doi.org/10.1111/j.1471-0528.2009.02279.x

[2] Ivars, J., Garabedian, C., Devos, P., Therby, D., Carlier, S., Deruelle, P., et al. (2016) Simplified Bishop Score Including Parity Predicts Successful Induction of Labor. European Journal of Obstetrics \& Gynecology and Reproductive Biology, 203, 309-314. https://doi.org/10.1016/j.ejogrb.2016.06.007

[3] Drgez, A., Girard, R., Dumas, A.-M., Mathiam, B. and Berland, M. (2008) Prediction of Successful Induction of Labor. Comparison between Bishop's Score and Fetal Fibronectin Dosage. Journal de Gynécologie Obstétrique et Biologie de la Reproduction, 37, 691-676. https://doi.org/10.1016/j.jgyn.2008.05.009

[4] Wing, D.A. and Lyons Gaffaney, C.A. (2006) Vaginal Misoprostol Administration for Cervical Ripening and Labor Induction. Clinical Obstetrics and Gynecology, 49, 627-641. https://doi.org/10.1097/00003081-200609000-00021

[5] Sherman, D.J., Frenkel, E., Pansky, M., Caspi, E., Bukovsky, I. and Langer, R. (2001) Balloon Cervical Ripening with Extra-Amniotic Infusion of Saline or Prostaglandin E2: A Double-Blind, Randomized Controlled Study. Obstetrics \& Gynecology, 97, 375-380. https://doi.org/10.1097/00006250-200103000-00010

[6] National Perinatal Survey: Report (2016) Births and settlements, Situation and Evolution Since 2010

http://www.epope/inserm.fr/wpcontent/uploads/2017/11/ENP2016_rapport_compl et.pdf

[7] Hale, R.W. and Zinberg, S. (2001) Use of Misoprostol in Pregnancy. New England Journal of Medicine, 344, 59-60. https://doi.org/10.1056/NEJM200101043440112

[8] Sangare, M. (2001) Induction of Labor in the Gynecology Obstetrics Department of the Point G Hospital Bamako (About 110 Cases). Med Thesis, Faculty of Medicine and Odontostomatolgy of Bamako, Bamako, 96 p, 56.

[9] Dolo, M. (2010) Artificial Induction of Labor: Use of Misoprostol in the Maternity Unit of the Commune VReferral Health Center. Med Thesis, Faculty of Medicine and Odontostomatolgy of Bamako, Bamako.

[10] Kouam, L., Kamdoum Mayo, J., Shasha, W. and Ngassa, P. (1993) Induced Labor: Conditions for Success and Causes of Failure, a Prospective Study of 162 Cases. $R e-$ vue Fr Gynecol Obstet, 88, 243-248.

[11] Bartusevicius, A., Barcaite, E. and Nadisauskiene, R. (2005) Oral, Vaginal and Sublingual Misoprostol For induction of Labor. International Journal of Gynecology \& Obstetrics, 91, 2-9. https://doi.org/10.1016/j.ijgo.2005.07.002

[12] Chiesa Moutandou-Mboumba, S., Mounanga, M. and Mayi, S. (1999) Artificial Induction of labor by Intra-Vaginal Misoprostol Prospective Study in Gabon in $97 \mathrm{~Pa}$ tients. Medicine of Black Africa.

[13] Ouerdiane, N., Tlili, N., Othmani, K., Daaloul, W., Masmoudi, A., Ben Hamouda, S. and Bouguerra, B. (2016) Onset of Term Labor by Misoprostol: Experience of a Tu- 
nisian Maternity Hospital. Pan African Medical Journal, 24, Article No. 28. https://doi.org/10.11604/pamj.2016.24.28.8141

[14] Pez, V., Deruelle, P., Kyheng, M., Boyon, C., Nailer, E. and Garabedian, C. (2018) Cervical Ripening Methods and Induction of Labor: Evaluation of the Single Balloon Catheter Compared to the Double Balloon Catheter and Prostaglandins. Gynécologie Obstétrique Fertilité \& Sénologie, 46, 570-574. https://doi.org/10.1016/j.gofs.2018.05.009

[15] Abbasi, N., Danish, N., Shakoor, F., Parveen, Z. and Ahmad Bilal, S. (2008) Effectiveness and Safety of Vaginal Misoprostol for Induction of Labor in a Favorable Cervix in $3^{\text {rd }}$ Trimester. Journal of Ayub Medical College Abbottabad, 20, 33-35.

[16] Diallo, D. (2009) Artificial Induction of Labor: Criteria Predicting Success and Maternal-Fetal Prognosis. Thesis DES Gynecology-Obstetrics, Faculty of Medicine and Odontostomatolgy of Bamako, Bamako. 